\title{
Ansiedad y depresión en pacientes posquirúrgicos en un hospital público de Lima
}

\author{
Anxiety and depression in post-surgical patients \\ in a public hospital in Lima \\ María del Rosario Mendoza Romero* \\ Escuela Profesional de Psicología, \\ Universidad de San Martín de Porres, Perú
}

\section{Resumen}

El objetivo del estudio fue establecer los niveles de ansiedad y depresión que presentan los pacientes que han sido sometidos a cirugía en un hospital público de Lima. Para ello se desarrolló una investigación de diseño no experimental y de alcance descriptivo, que se trabajó con una muestra intencionada de 110 pacientes intervenidos quirúrgicamente. Por su propia condición, debieron absolver un cuestionario relativamente corto, basado en la Escala de Ansiedad y Depresión Hospitalaria (HADS). Los resultados muestran que los niveles de ansiedad en estos pacientes son relativamente bajos (62.7\% del total), atribuibles a la situación quirúrgica que están viviendo; ocurriendo algo similar en relación con los niveles de depresión, que también son relativamente bajos $(78.2 \%$ del total). Sin embargo, es necesario ponerle atención a quienes se encuentran en un nivel medio de depresión (11.8\% del total) y en un nivel bajo (10\% del total), por los problemas que ello podría ocasionar.

Palabras clave: ansiedad, depresión, angustia, tristeza, dolor. 


\begin{abstract}
The objective of the study was to establish the levels of anxiety and depression presented by patients who have undergone surgery in a public hospital in Lima. For this, a non-experimental design and descriptive research was developed, which was worked with a purposive sample of 110 patients who underwent surgery. Due to their own condition, they had to complete a relatively short questionnaire based on the Hospital Anxiety and Depression Scale (HADS). The results show that anxiety levels in these patients are relatively low $(62.7 \%$ of the total), attributable to the surgical situation they are experiencing; something similar happening in relation to depression levels, which are also relatively low (78.2\% of the total). However, it is necessary to pay attention to those who are in a medium level of depression (11.8\% of the total) and in a low level (10\% of the total), due to the problems that this could cause.
\end{abstract}

Keywords: anxiety, depression, anguish, sadness, hurt.

\title{
Introducción
}

Un informe publicado por la Organización Mundial de la Salud [OMS] (2017) indica que, en el ámbito global, los casos de ansiedad y depresión continúan en ascenso. El 12\% de la población europea presenta depresión y el 14\% registra ansiedad; en ambos porcentajes la mayor prevalencia corresponde a las mujeres. En las Américas, el 15\% de la población sufre trastornos depresivos y el $21 \%$ trastornos de ansiedad. Igual que en Europa, las mujeres muestran mayores índices de ansiedad y depresión que los varones.

En nuestro país, de acuerdo con lo reportado por el Ministerio de Salud [MINSA] (2016), los problemas de salud mental son importantes, pudiendo afectar al 20\% de la población adulta. Dentro de ellos destacan los trastornos de ansiedad y depresión, siendo Lima una de las ciudades con mayor incidencia de este problema al presentar un $16.4 \%$ de casos de depresión, seguidos por el trastorno de ansiedad generalizada con un 10.6\% (Instituto 
Nacional de Salud Mental Honorio Delgado - Hideyo Noguchi [INSM], 2021). Es verdad que, en el Perú, la prioridad de la atención en salud se dirige a las afecciones físicas, en tanto se pretende reducir los porcentajes de mortalidad. Por ello, los trastornos de salud mental, como la ansiedad y la depresión, no son lo suficientemente atendidos y menos cuando se trata de pacientes quirúrgicos.

En tal contexto, se estima que estos pacientes -que demandan cuidados especializados en las salas de cirugía-, debido a los eventos por los cuales deben pasar, requieren necesariamente de apoyo psicológico para manejar y/o reducir sus niveles de ansiedad y, eventualmente, de depresión. Se ha visto que la terapia psicológica logra efectos positivos en su afrontamiento de la enfermedad, en su preparación preoperatoria y en su recuperación posoperatoria (Remor et al., 2010; Uman et al., 2013). De acuerdo con Spinelli et al. (2014), si se diseña un adecuado plan prequirúrgico que incluya asistencia psicológica, se obtendrán resultados satisfactorios al reducirse sensiblemente cualquier tipo de emociones negativas que pudieran surgir.

Los problemas de posible aparición en estos pacientes se encuentran relacionados con un conjunto de estímulos y estados de índole negativa que, sin duda, van a impedir una correcta evolución posoperatoria, la adhesión al tratamiento y la propia recuperación, lo que podría traer como consecuencia la ocurrencia de recaídas, con los consiguientes perjuicios que ello supone, al incrementarse la frecuencia de hospitalizaciones (Anguiano et al., 2017). Se debe considerar, por ejemplo, que episodios ansiosos pueden menoscabar la resistencia inmunológica, mientras que la depresión perjudicará el proceso de recuperación. De allí la importancia de la intervención del psicólogo, en la medida en que es capaz de detectar a tiempo estas condiciones emocionales del paciente y trabajar sobre ello.

Es probable que sean estas razones las que han impulsado, en los últimos tiempos, el desarrollo de un conjunto de investigaciones que vinculan los estados de ansiedad y depresión con la salud física, particularmente en pacientes con alguna dolencia de este tipo. Dichas investigaciones han permitido incrementar el acervo de conocimientos sobre estas dificultades, permitiendo una mejor comprensión respecto de los estados emocionales 
de los pacientes hospitalizados en general, y de los pacientes posquirúrgicos en particular. Sin embargo, se requiere un mayor número de estudios (Cano, 2011) y el fomento de programas de intervención que reduzcan los problemas mencionados.

No se puede dejar de considerar que en estos pacientes la ansiedad puede alcanzar niveles patológicos. De acuerdo con lo señalado por el Ministerio de Sanidad, Consumo y Bienestar Social [MSCBS] de España (2016), la ansiedad es parte de la respuesta del ser humano frente a un eventual peligro, por lo que es preciso adoptar las medidas necesarias para lidiar con este problema. No obstante, si la ansiedad supera las barreras desarrolladas por el ser humano, se convierte en patológica y esto sí afecta seriamente su salud física y mental.

Por su parte, Fernández et al. (2009) consideran que la depresión, siendo un trastorno grave, no recibe regularmente el tratamiento de alivio correspondiente, y sus consecuencias afectan la vida diaria tanto de quienes la padecen como de sus familias. Es cierto que cuando la depresión es leve, tiende a desaparecer con el transcurso de los días, lo que abona en favor de asegurar un tratamiento adecuado.

El propósito de la presente investigación fue establecer los niveles de ansiedad y depresión en pacientes posquirúrgicos, razón por la cual se plantearon las siguientes interrogantes:

1. ¿Cuáles son los niveles de ansiedad que se presentan en pacientes posquirúrgicos de un hospital público de Lima?

2. ¿Cuáles son los niveles de depresión que se presentan en pacientes posquirúrgicos de un hospital público de Lima?

3. ¿Cuál es el nivel de ansiedad, según sexo, que se presenta en los pacientes posquirúrgicos de un hospital público de Lima?

4. ¿Cuál es el nivel de depresión, según sexo, que se presenta en los pacientes posquirúrgicos de un hospital público de Lima? 


\section{Objetivos}

1. Describir los niveles de ansiedad que se presentan en pacientes posquirúrgicos de un hospital público de Lima.

2. Identificar los niveles de depresión que se presentan en pacientes posquirúrgicos de un hospital público de Lima.

3. Describir el nivel de ansiedad, según sexo, que se presenta en los pacientes posquirúrgicos de un hospital público de Lima.

4. Identificar el nivel de depresión, según sexo, que se presenta en los pacientes posquirúrgicos de un hospital público de Lima.

\section{Método}

El método de investigación utilizado fue el descriptivo. Para Sánchez y Reyes (2006), este método permite la descripción de las diversas características de un fenómeno en un determinado momento en el tiempo, con el propósito de lograr especificar cuáles son las propiedades que lo identifican.

\section{Diseño de investigación}

Se trata de un diseño no experimental de nivel descriptivo, de acuerdo con lo propuesto por Hernández et al. (2014), ya que su propósito es describir las variables individuales y cómo se comportan en un determinado momento en el tiempo.

\section{Muestra}

El establecimiento de la muestra, siguiendo a Hernández et al. (2014), es no probabilístico de tipo intencionado, dado que se consideró a pacientes intervenidos quirúrgicamente que estaban en condiciones de responder al cuestionario y que dieran su consentimiento para hacerlo. El total de pacientes que aceptó y resolvió los cuestionarios fue de 110. 
Tabla 1

Distribución de la muestra por sexo

\begin{tabular}{ccc}
\hline Sexo & Frecuencia & Porcentaje (\%) \\
\hline Varón & 43 & 39.1 \\
Mujer & 67 & 60.9 \\
\hline Total & 110 & 100.0 \\
\hline
\end{tabular}

Los resultados que se exponen en la tabla 1 informan que las mujeres son mayoría en la muestra al alcanzar el $60.9 \%$, mientras que los varones representan el $39.1 \%$.

\section{Instrumentos}

En la presente investigación, como instrumento de recolección de datos se utilizó la Escala de Ansiedad y Depresión Hospitalaria (HADS) de Zigmond y Snaith (1983), conformada por 14 ítems, distribuidos en dos subescalas de siete ítems cada una: ansiedad (reactivos impares) y depresión (reactivos pares).

\section{Análisis de validez y confiabilidad del HADS}

Este instrumento fue sometido a los respectivos análisis que permitieran garantizar su validez y confiabilidad. Para el efecto, los datos recolectados fueron llevados a una base de datos del paquete estadístico SPSS versión 27. Por la naturaleza del instrumento, este fue procesado como una escala total $(\alpha=.88)$, para después separar las dos subescalas. En principio se evaluó la parte de ansiedad, la que fue sometida al estadístico de confiabilidad alfa de Cronbach, dando un valor de .79. La subescala de depresión fue sometida al mismo estadístico, obteniéndose un valor de .75 . Estos resultados permiten determinar que la escala es confiable en sus dos subescalas.

En lo que respecta a la validez, las evaluaciones se realizaron con el apoyo del análisis factorial exploratorio, cuyos resultados muestran valores lo suficientemente altos como para comprobar la validez de la escala HADS en sus dos dimensiones. Para la calificación de la prueba se utilizaron los puntos de corte formulados por López-Alvarenga et al. (2002), quienes establecieron que en cuanto a ansiedad el punto de corte se ubicaba en 
puntajes iguales o mayores a los ocho puntos, y para la depresión en puntajes iguales o mayores a siete puntos.

\section{Resultados}

\section{Análisis descriptivos}

\section{Tabla 2}

Niveles de ansiedad

\begin{tabular}{lcc}
\hline Niveles & Frecuencias & Porcentajes (\%) \\
\hline Bajo & 69 & 62.7 \\
Medio & 30 & 27.3 \\
Alto & 11 & 10.0 \\
\hline Total & 110 & 100.0 \\
\hline
\end{tabular}

\section{Figura 1}

Niveles de ansiedad en la muestra tomada

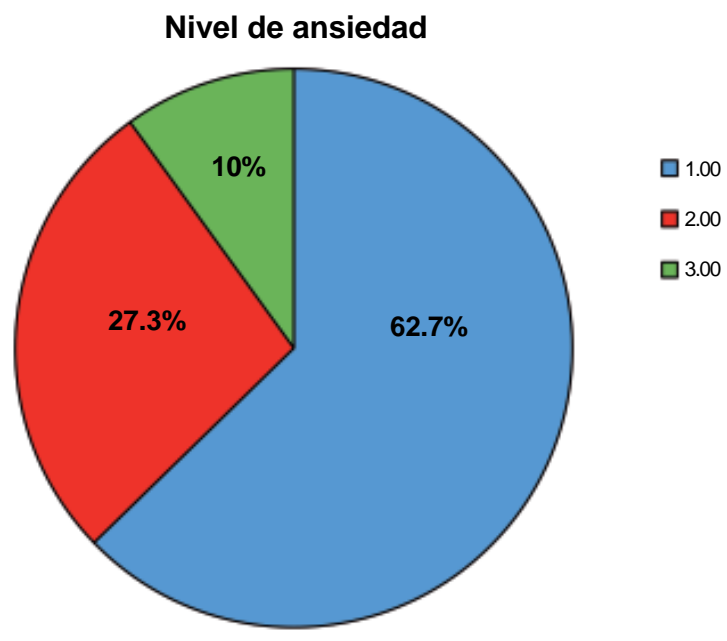

Los resultados obtenidos en la Tabla 2 y en la Figura 1 reportan que los niveles de ansiedad en la muestra tomada se ubican de la siguiente manera: el $62.7 \%$ se encuentra en un nivel bajo, el $27.3 \%$ en un nivel medio y el $10 \%$ en un nivel alto. 


\section{Tabla 3}

Niveles de depresión

\begin{tabular}{lcc}
\hline Niveles & Frecuencias & Porcentajes (\%) \\
\hline Bajo & 86 & 78.2 \\
Medio & 13 & 11.8 \\
Alto & 11 & 10.0 \\
\hline Total & 110 & 100.0 \\
\hline
\end{tabular}

\section{Figura 2}

Niveles de depresión en la muestra tomada

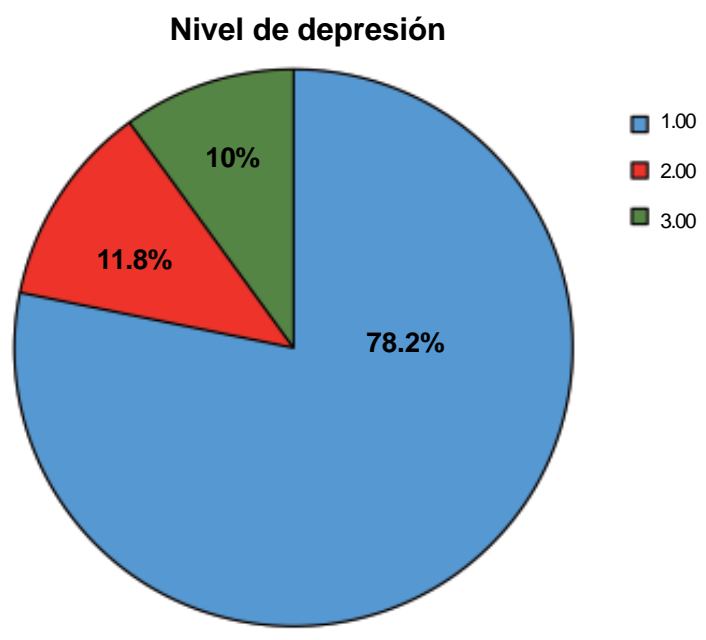

Los resultados obtenidos en la Tabla 3 y en la Figura 2 reportan que los niveles de depresión en la muestra tomada se ubican de la siguiente manera: el $78.2 \%$ se encuentra en un nivel bajo, el $11.8 \%$ en un nivel medio y el $10 \%$ en un nivel alto.

Tabla 4

Niveles de ansiedad según sexo

\begin{tabular}{ccc}
\hline Sexo & Frecuencias & Porcentajes (\%) \\
\hline Varón & 5 & 45.50 \\
Mujer & 6 & 54.50 \\
\hline Total & 11 & 100.00 \\
\hline
\end{tabular}


Los resultados obtenidos en la Tabla 4 reportan que el nivel de ansiedad según sexo en la muestra tomada se ubica de la siguiente manera: el $45.50 \%$ corresponde a los varones y el $54.50 \%$ corresponde a las mujeres.

\section{Tabla 5}

Niveles de depresión según sexo

\begin{tabular}{ccc}
\hline Sexo & Frecuencias & Porcentajes (\%) \\
\hline Varón & 4 & 36.40 \\
Mujer & 7 & 63.60 \\
\hline Total & 11 & 100.00 \\
\hline
\end{tabular}

Los resultados obtenidos en la Tabla 5 reportan que el nivel de depresión según sexo en la muestra tomada se ubica de la siguiente manera: el $36.40 \%$ corresponde a los varones y el $63.60 \%$ corresponde a las mujeres.

\section{Discusión}

En principio, y dadas las exigencias de que el instrumento empleado contara con los niveles de validez y confiabilidad necesarios para garantizar la utilidad de la prueba, se realizaron los procedimientos estadísticos orientados a lograr tal fin. Así tenemos que la Escala de Ansiedad y Depresión Hospitalaria (HADS) fue procesada atendiendo al total de la escala $(\alpha=.88)$ y a cada una de sus dos subescalas (ansiedad y depresión), dando como resultado que ambas son válidas y confiables, tal cual se desprende de los valores que alcanzaron tanto en el coeficiente alfa de Cronbach como en el análisis factorial exploratorio.

En lo que se refiere a los objetivos planteados, destinados a establecer los niveles de ansiedad y depresión que se presentan en los pacientes posquirúrgicos, los resultados informan que los niveles de ansiedad son relativamente bajos $(62.7 \%)$, en tanto existen preocupantes niveles medios $(27.3 \%)$ y altos (10\%) que deben llamar la atención sobre la necesidad de atender estos casos con urgencia. Por el lado de la depresión los resultados se encuentran prácticamente en la misma línea, pues alcanzaron valores que llegan al 78.2\% para ubicar los casos bajos de depresión, $11.8 \%$ para los niveles medios y $10 \%$ para los niveles altos. Sin duda, es preciso que el 
trabajo del psicólogo pueda ser más ampliamente desarrollado en estas áreas, de manera que se pueda prevenir y/o minimizar los casos reportados de ansiedad y depresión en este tipo de pacientes.

Por otra parte, resulta importante señalar que los resultados obtenidos se pueden contrastar con los que obtuvieron autores como Borges (2018), Guillén y Ordóñez (2018) y Cabrera et al. (2015), quienes coinciden en mostrar que la Escala de Ansiedad y Depresión Hospitalaria (HADS) alcanza importantes niveles de confiabilidad y validez. Por otra parte, encuentran también que los pacientes hospitalizados presentan síntomas de ansiedad y depresión en distintos niveles. Esto se puede explicar atendiendo al hecho de que las condiciones hospitalarias, tanto en términos de infraestructura como en lo que respecta a la calidad de la atención, no son exactamente las mejores para los pacientes, por lo que se considera casi «normal» que la ansiedad esté presente.

Se debe tener en cuenta que los estados emocionales incluyen una variedad grande de condiciones que van a afectar significativamente la capacidad de los pacientes para manejar las demandas que deben enfrentar, pudiéndose presentar dificultades en el pensamiento, en los sentimientos y en las relaciones personales. Por otro lado, cabe enfatizar que la ansiedad y la depresión son trastornos que regularmente van acompañados; es decir que cualquier persona con síntomas depresivos puede tener fácilmente síntomas de ansiedad y viceversa (Teva et al., 2005).

En la presente investigación se ha encontrado también que niveles altos de ansiedad y depresión son más numerosos en las pacientes mujeres, lo que se vincularía con los resultados de Abello et al. (2014), quienes concluyen que las mujeres presentan un mayor nivel de sintomatología depresiva que los varones. Sin embargo, hay otros estudios como el de Vinaccia et al. (2006), quienes señalan que los varones registran mayores niveles de depresión que las mujeres.

Resulta importante mencionar que diversos estudios reportan el hecho de que la atención psicológica antes y después de la cirugía tiene resultados 
positivos. En Cuba, por ejemplo, Nápoles et al. (2013) demostraron que, empleando técnicas de relajación frente a la proximidad de cirugía de mama por cáncer, se logró disminuir la ansiedad de manera significativa. Por otra parte, trabajos como los de Kain et al. (2007) han demostrado que la terapia cognitivo-conductual ha sido efectiva para reducir los niveles de ansiedad. Del mismo modo, se han reportado resultados exitosos de las técnicas conductuales (Uman et al., 2006), mientras que el mindfulness ha logrado mejorar la calidad de vida de los pacientes (Nyklicek et al., 2014).

Finalmente se debe señalar que los hallazgos aquí presentados llevan a concluir que las propiedades de confiabilidad y validez del HADS para pacientes posquirúrgicos son adecuadas, además de la ventaja adicional otorgada por la brevedad de la escala, lo que facilita su aplicación. Por último, se sugiere que futuras investigaciones amplíen la muestra de manera que se puedan realizar los análisis factoriales confirmatorios correspondientes.

\section{Conclusiones}

Todo lo abordado es de vital importancia para el profesional de la psicología. Los resultados alcanzados nos indican que los trastornos de ansiedad en los pacientes posquirúrgicos de un hospital público de Lima se ubican preferentemente en un nivel bajo, igual que los trastornos por depresión. Considerando el sexo de los sujetos de la muestra, las mujeres obtienen porcentajes más altos que los varones tanto en lo que se refiere a ansiedad como a depresión.

Es necesario que el trabajo del psicólogo sea más ampliamente desarrollado en estas áreas, de manera que se puedan prevenir y/o minimizar los casos reportados de ansiedad y depresión en este tipo de pacientes. 


\section{Referencias}

Abello, D., Cortés, O. F., Barros, O., Osorio, S. y Solano, D. (2014). Prevalencia de depresión infantil en colegios oficiales de Barranquilla. Tesis Psicológica, 9(1), 190-201. http://www.redalyc.org/pdf/1390/139031679015.pdf

Anguiano, S. A., Mora, M. A., Reynoso, L. y Vega. C. Z. (2017). Prevalencia de ansiedad y depresión en pacientes hospitalizados. Eureka, 14(1), 24-38. https://docs.bvsalud.org/biblioref/2018/06/905744/eureka-14-19.pdf

Borges, T. (2018). Estudio sobre la depresión y ansiedad mediante el PHQ en Venezuela [Tesis doctoral, Universidad Complutense de Madrid]. https://eprints.ucm.es/id/eprint/46763/1/T39658.pdf

Cabrera, V., Martín-Aragón, M., Terol, M., Núñez, R. y Pastor, M. (2015). La Escala de Ansiedad y Depresión Hospitalaria (HADS) en fibromialgia: Análisis de sensibilidad y especificidad. Terapia Psicológica, 33(3), 181193. https://dx.doi.org/10.4067/S0718-48082015000300003

Cano, A. (2011). Bases teóricas y apoyo empírico de la intervención psicológica sobre los desórdenes emocionales en atención primaria. Una actualización. Revista Ansiedad y Estrés, 17(2-3), 157-184.

Fernández, P., Moreno, A., Leza, J., Lizasoain, I., Moro, M. y Portolés, A. (2009). Farmacología básica y clínica (19. ${ }^{\mathrm{a}}$ ed.). Editorial Médica Panamericana.

Guillén, J. y Ordóñez, R. (2018). Depresión y ansiedad en pacientes adultos en hemodiálisis de un hospital nacional de la Policía Nacional del Perú [Tesis de Licenciatura, Universidad Ricardo Palma]. https://repositorio.urp.edu.pe/ bitstream/handle/URP/1199/TESIS-Guillen-Ordonez.pdf?sequence=1\&isAllowed=y

Hernández, R., Fernández, C. y Baptista, P. (2014). Metodología de la investigación. Mc Graw Hill.

Instituto Nacional de Salud Mental Honorio Delgado - Hideyo Noguchi (INSM). (2021). Nota de prensa 13/12. http://www.insm.gob.pe/OFICINAS/COMUNICACIONES/notasdeprensa/2013/012.html

Kain, Z. N., Caldwell-Andrews, A. A., Mayes, L. C., Weinberg, M. E., Wang, S. M., MacLaren, J. E. y Blount, R. L. (2007). Family-Centered Preparation for Surgery Improves Perioperative Outcomes in Children: A Randomized Controlled Trial. Anesthesiology, 106(1), 65-74.

López-Alvarenga, J. C., Vázquez-Velázquez, V., Arcila-Martínez, D., Sierra-Ovando, A. E., González-Barranco, J. y Salín-Pascual, R. (2002). Exactitud y utilidad diagnóstica del Hospital Anxiety and Depression Scale (HADS) en una muestra de sujetos obesos mexicanos. Revista de Investigación Clínica, 54(5), 403-409.

Ministerio de Salud - MINSA. (2016). Ley No 26842. Ley general de salud. https://www.digemid.minsa.gob.pe/ upload/uploaded/pdf/leyn26842.pdf

Ministerio de Sanidad, Consumo y Bienestar Social - MSCBS. (2016). Guía de práctica clínica para el manejo de pacientes con trastornos de ansiedad en atención primaria de salud. Ed. Agencia Laín Entralgo. Unidad de Evaluación de Tecnologías Sanitarias.

Nápoles, Y., Agüero, M., Díaz, H. y Espino, R. M. (2013). Aplicación de técnicas de relajación antes de cirugía de la mama. Archivo Médico de Camagüey, 17(2), 173-186.

Nyklicek, I., Dijksman, S. C., Lenders, P. J., Fontejin, W. A. y Koolen, J. J. (2014). A brief mindfulness based intervention for increase in emotional well-being and quality of life in percutaneous coronary intervention (PCl) patients: The MindfulHeart randomized controlled trial. Journal Behaviour Medicine, 37(1), 135-144. doi: 10.1007/ s10865-012-9475-4.

Organización Mundial de la Salud - OMS. (2017). Cifras y datos 2017. http://bit.ly/2oYBFAv

Remor, E., Amorós, M. y Carrobles, J. A. (2010). Eficacia de un programa manualizado de intervención en grupo para la potencialización de las fortalezas y recursos psicológicos. Anales de Psicología, 26(1), 49-57. 
Sánchez, H. y Reyes, C. (2006). Metodología y diseño de la investigación científica. Editorial Visión Universitaria.

Spinelli, A., Allocca, M., Jovani, M. y Danese, S. (2014). Optimal preparation for surgery in Crohn's disease. Alimentary Pharmacology and Therapeutics, 40(9), 1009-1022. https://onlinelibrary.wiley.com/doi/pdfdirect/10.1111/ apt.12947

Teva, I., Bermúdez, M. P., Hernández-Quero, J. y Buela-Casal, G. (2005). Evaluación de la depresión, ansiedad e ira en pacientes con VIH/SIDA. Salud Mental, 28(5), 40-49. https://www.redalyc.org/pdf/582/58252806.pdf

Uman, L. S., Birnie, K. A., Noel, M., Parker, J. A., Chambers, C. T., Mcgrath, P. J. y Kiseley, S. R. (2013). Psychological interventions for needle-related procedural pain and distress in children and adolescents. Cochrane Database System Review, 10(10), 1-137. doi: 10.1002/146558.

Uman, L. S., Chambers, C. T., Mcgrath, P. J. y Kiseley, S. R. (2006). Psychological Interventions for NeedleRelated Procedural Pain and Distress in Children and Adolescents. Cochrane Database System Review, 18(4), 1-135. doi: 10.1002/14651858. CD005179.pub2

Vinaccia, S., Gaviria, A. M., Atehortúa, L. F., Martínez, P. H., Trujillo, C. y Quinceno, J. M. (2006). Prevalencia de depresión en niños escolarizados entre 8 y 12 años del oriente antioqueño a partir del «child depression inventory» - CDI. Diversitas: Perspectivas en Psicología, 2(2), 217-227. https://www.redalyc.org/pdf/679/ 67920203.pdf

Zigmond, A. S. y Snaith, R. P. (1983). The Hospital Anxiety and Depression Scale. Acta Psychiatrica Scandinavica, 67, 361-370. 